\title{
Coletivo pensante seres-humanos-com-geogebra-e-smartphone: demonstrando a fórmula de Bhaskara
}

\author{
Thaynara Menezes Gandra Conceição ${ }^{1}$ \\ Eder Marinho Martins ${ }^{2}$ \\ Wenderson Marques Ferreira ${ }^{3}$ \\ Edmilson Minoru Torisu ${ }^{4}$
}

\section{Resumo}

Tecnologias digitais estão presentes em nossas vidas e isso muda de forma importante a forma como nos comunicamos e aprendemos. Nas escolas, essas tecnologias podem ser utilizadas como mediadores da aprendizagem, possibilitando aos estudantes a apropriação de novos conteúdos, ou a ressignificação de conteúdos já aprendidos. Esse estudo teve por objetivo desvelar apropriaçóes de novos conhecimentos por parte de estudantes de uma escola privada da cidade de Belo Horizonte, Minas Gerais, Brasil, quando participaram como atores de um coletivo pensante seres-humanos-com-GeoGebra-e-smartphone. Os dados foram obtidos por meio de observaçóes realizadas durante uma atividade que tinha como objetivo a demonstração da fórmula de Bhaskara com o uso do GeoGebra, por meio de um questionário. Os resultados mostraram que novos conhecimentos foram apropriados e revelaram a importância da compreensão da aprendizagem como resultado das interações entre os atores humanos e não humanos de um coletivo.

Palavras-chave: fórmula de Bhaskara; seres-humanos-com-GeoGebra-e-smartphone; apropriação.

1 Licenciada em Matemática pela Universidade Federal de Minas Gerais, Mestra em Matemática pelo Mestrado Profissional em Matemática em Rede Nacional - PROFMAT/UFOP. Professora da rede privada de educação básica de Belo Horizonte - MG. E-mail: thaynaragandra@hotmail.com.

2 Doutor em Matemática pela Universidade Federal de Minas Gerais, Professor Associado do Departamento de Matemática da Universidade Federal de Ouro Preto, Professor do Mestrado Profissional em Matemática em Rede Nacional - PROFMAT. E-mail: eder@ufop.edu.br.

3 Doutor em Matemática pela Universidade Federal de Minas Gerais, Professor Associado do Departamento de Matemática da Universidade Federal de Ouro Preto, Professor do Mestrado Profissional em Matemática em Rede Nacional - PROFMAT. E-mail: wmf@ufop.edu.br.

4 Mestre em Educação Matemática pela Universidade Federal de Ouro Preto, Doutor em Educação pela Universidade Federal de Minas Gerais, Professor Adjunto de Departamento de Educação Matemática da Universidade Federal de Ouro Preto e Professor do Programa de Pós-Graduação em Educação Matemática da mesma instituição. E-mail: edmilson@ufop.edu.br. 
Human--with-geogebra-and-smartphone collective thinking: Demonstrating the
quadratic formula

\section{Abstract}

Digital technologies are present in our lives and this changes significantly the way we communicate and learn. In schools, these technologies can be used as mediators of learning enabling students to appropriate new content or to give a new significate to previously studied ones. The purpose of this study was to uncover appropriations of new knowledge by students of a private school in the city of Belo Horizonte, Minas Gerais, Brazil, when they participated as actors of a humans-with-GeoGebra-and-smartphonecollective thinking. The data were obtained through observations made during an activity, whose objective was to demonstrate the Quadratic Formula with the use of GeoGebra, and through a questionnaire. The results indicated that new knowledge was appropriate and revealed the importance of learning comprehension as a result of the interactions between humans and non human actors of a collective.

Keywords: quadratic formula; Humans-with-GeoGebra-and-smartphone; appropriation.

\section{Introduçáo}

As tecnologias digitais estão cada vez mais presentes em nossas vidas, modificando-as. Muitas das necessidades do mundo globalizado (do qual fazemos parte) são satisfeitas por avanços dessas tecnologias. Uma relação simbiótica parece ter se estabelecido entre tecnologias e mundo globalizado.

Particularmente no ensino, o uso das tecnologias digitais tem permitido novas possibilidades para os modos de aprendizagem das pessoas. Hoje, aprende-se com jogos digitas, softwares interativos de interface amigável, à distância, pelo smartphone, dentre outras possibilidades. Todas elas permeadas pelas tecnologias digitais.

As tecnologias digitais foram se sofisticando e, na perspectiva de Borba, Scucuglia e Gadanidis (2014), seu desenvolvimento envolve algumas fases. Na primeira fase, iniciada no final da década de 1980, acreditava-se que o computador seria o catalisador de mudanças pedagógicas. O software Logo é bastante representativo dessa fase. A segunda fase, que teve início em meados de 1990, é marcada pela popularização dos chamados personal computers, surgimento de softwares de interface amigável a aumento dos cursos de formação de professores. Por volta de 1999 inicia-se a terceira fase, marcada pelos cursos a distância on line. A quarta fase inicia-se por volta de 2004 
com o advento da internet de alta velocidade. Como exemplo de softwares importantes que marcaram (marcam) essa fase, podemos citar o GeoGebra.

A apresentação, ainda que resumida, dessas fases, mostra como as tecnologias digitais podem ter impactado a maneira de aprender das pessoas. Seu uso permite que elas se apropriem de novos conhecimentos ou ressignifiquem outros a partir da reorganização do pensamento (THIKOMIROV, 1981). Na próxima seção discutiremos um pouco mais essa ideia.

\section{Tecnologias, apropriação e reorganização do pensamento}

De acordo com Veraszto et al. (2008), a palavra tecnologia provém de uma junção do termo tecno, do grego techné, que é saber fazer, e logia, do grego logus, que significa razão, ou seja, tecnologia significa a razão do saber fazer. É o estudo da técnica, da própria atividade do modificar, do transformar, do agir.

A despeito dessa definição ampliada do termo, a palavra tecnologia tem sido associada, sobretudo em tempos atuais, a computadores, internet, jogos digitais, redes sociais, softwares, smartphones, etc. Quando o assunto é o uso de tecnologias no ensino, somos sempre tentados a fazer as mesmas associaçóes. Dessa forma, lápis e papel seriam considerados ultrapassados e nada tecnológicos. Lápis e papel, entretanto, foram, e ainda são, uma técnica que modificou/modifica o conhecimento de todos nós. Portanto, são uma forma de tecnologia. Elas sempre estiveram presentes na história da humanidade refletindo determinado momento histórico.

Pierre Levy (1993) considera três técnicas associadas às tecnologias da inteligência, ligadas à memória e ao conhecimento: a oralidade, a escrita e a informática. Antes do advento da escrita, a oralidade era a tecnologia mais importante para que as pessoas adquirissem conhecimento. A escrita estendeu qualitativamente a memória, pois permitiu o surgimento da linearidade do raciocínio. A informática, por sua vez, avança no que se refere à extensão da memória, já que alia oralidade, escrita, imagens, experimentação e visualização, desafiando a linearidade do pensamento. Ou seja, o uso de diferentes tecnologias permite um salto qualitativo no processo de extensáo da memória. É importante, porém, que não entendamos a construção do conhecimento por meio de tecnologias como uma via de mão única, como se as pessoas fossem apenas receptoras passivas, numa visão quase behaviorista da aprendizagem. Da mesma forma que as mídias estendem e modificam 
o raciocínio, as pessoas estão constantemente transformando essas mídias, numa relação dialética.

Em se tratando de tecnologias digitais, sobretudo dos computadores e smartphones, é inegável que têm sido amplamente utilizados em vários ramos da sociedade e que mudaram, sobremaneira, a forma como as pessoas se comunicam, interagem e aprendem. Existe, portanto, uma íntima relação entre o uso de computadores e a cognição humana.

Tikhomirov (1981) apresenta três teorias para explicar esta relação. $\mathrm{Na}$ primeira, o computador seria um substituto das atividades intelectuais do homem. O autor critica essa teoria ao explicar que homem e máquina utilizam processos diferentes para resolver o mesmo problema e que, portanto, não podemos falar de substituição. Além disso, embora essa ideia não seja atribuída ao citado autor, considerar que o computador pode substituir o homem é subestimar a capacidade cognitiva de quem o criou. A segunda teoria defende que o computador suplementa o pensamento em termos quantitativos, desconsiderando o aspecto qualitativo da relação. Esta teoria também é rejeitada por Tikhomirov. Ele considera como mais adequada a teoria que defende o computador (e poderíamos acrescentar smartphones, tablets, etc) como uma ferramenta de mediação entre o homem e o saber, reorganizando o pensamento.

A ideia de mediação, central na terceira teoria, é o cerne da teoria vygotskiana. De forma ampliada, a mediação "é toda a intervenção de um terceiro 'elemento' que possibilita a interação entre os 'termos' de uma relação" (PINO, 1991). Esse elemento mediador pode ser de dois tipos: instrumentos e signos.

enquanto o signo constitui uma atividade interna dirigida para o controle do próprio sujeito, o instrumento é orientado externamente para o controle da natureza. Tanto o controle do comportamento como o da natureza acarretam mudanças no funcionamento cognitivo, o primeiro ocasionando a emergência das funçôes superiores e o segundo a relação do homem com o seu ambiente: o homem muda a natureza e essa mudança altera a sua própria natureza. (VIGOTSKY, 1978, apud RIPPER, 1993, p. 25).

A partir das ideias acima, os computadores, smartphones e outras tecnologias digitais, quando se interpóem entre estudante e o objeto de estudo, podem funcionar como mediadores do processo de aprendizagem e provocar mudanças cognitivas. Tais mudanças talvez possam ser compre- 
endidas em termos de saltos qualitativos, como consequência da reorganização do pensamento.

Ao introduzir as tecnologias digitais para ensinar na sala de aula estamos introduzindo algo familiar aos estudantes, mas não naquele contexto. Computadores e smartphones, na maioria das vezes, não são instrumentos amplamente utilizados pelos professores com o objetivo de ensinar em sala de aula. O estudante, quando em contato com essa proposta, acaba por apropriar-se dessa nova forma de uso. Apropriaçáo aqui, mais uma vez, tem raízes na teoria histórico-cultural e "refere-se a modos de tornar próprio, de tornar seu" (SMOLKA, 2000, p. 28). O computador ou smartphone que, pelo processo de objetivação, encarna uma história de criação e seus usos passam a ter, para os estudantes, após o processo de apropriação, um outro significado: o de instrumentos de aprendizagem. Essa apropriação ocorre no interior das relaçóes sociais (professores, colegas, sala de aula) e os conhecimentos apropriados são reelaborados num plano pessoal interno (internalização), que resulta no desenvolvimento de novas funções mentais. Em outras palavras, "(o) processo de internalização transforma o social em subjetivo e isso é uma nova produção" (TORISU, 2018). Isso faz sentido quando lembramos do que nos aponta Vygotsky (1989, p. 56): "as funçóes mentais superiores são relações sociais internalizadas” e reelaboradas.

As experiências sociais, entretanto, com essas tecnologias digitais, não se restringem apenas a reconhecê-las como instrumentos para aprendizagem. As experiências englobam o seu uso prático quando utilizamos, por exemplo, um software de geometria dinâmica para explorar funçóes. Recursos como imagens, manipulação e visualização permitem ao estudante acessar o objeto matemático (nesse caso) de formas diferentes das quais ele está acostumado. Ao se apropriar dessas novas formas de "ver" o objeto matemático e o próprio computador, o estudante, por um processo de internalização, transforma o vivido no social em novo conhecimento, digamos, reorganizado. Contudo, o vivido no social não se restringe ao conteúdo. Ele é um pacote que envolve o conteúdo a ser aprendido, o computador ou tecnologia digital presente, o professor e os colegas num processo interativo e coletivo que pode resultar na produção do conhecimento. Tikhomirov (1981) denomina esse coletivo de ser-humano-computador. Mais tarde, Borba e Vilarreal (2005) ampliam essa ideia e propóem a metáfora seres-humanos-com-mídias, que passa a contemplar outras mídias, como lápis e papel, por exemplo, como atores informáticos (BORBA, 2001) que contribuem para a construção/reorganização do conhecimento. 
Neste texto, apresentamos uma proposta para demonstração da fórmula de Bhaskara utilizando o software GeoGebra. A fórmula de Bhaskara é comumente apresentada aos estudantes no nono ano do Ensino Fundamental, por ocasião do estudo das equaçóes de segundo grau. A maioria deles, entretanto, não sabe de onde ela surge e contenta-se em decorá-la. A escolha pelo GeoGebra se justifica pelo fato de ser um software de interface amigável e com muitos recursos, além de ser uma ferramenta nova para os estudantes. Neste coletivo seres-humanos-com-GeoGebra-e-smartphone, o acesso ao conteúdo foi mediado por ferramentas e signos num processo que levou à apropriação de novos modos de fazer num processo que pode ter levado à reorganização do pensamento.

\section{Sujeitos e contexto}

Esta pesquisa, de natureza qualitativa, teve como objetivo desvelar apropriaçóes de novos conhecimentos relacionados à demonstração da fórmula de Bhaskara num coletivo seres-humanos-com-GeoGebra-e-smartphone e apresentar indícios de pensamento reorganizado.

Os sujeitos deste estudo foram vinte e oito alunos de uma escola privada da região noroeste de Belo Horizonte, Minas Gerais, Brasil. Esses alunos estavam cursando o nono ano do ensino fundamental e já haviam estudado o conteúdo de funçóes quadráticas com seus respectivos professores de Matemática.

A produção de resultados, de natureza qualitativa, foi fundamentada no comportamento e fala dos alunos durante a aplicação da atividade e na utilização de questionários.

A atividade permitiu que os alunos fizessem a demonstração da fórmula de Bhaskara sem completar quadrados, o que é de difícil compreensão para grande parte dos estudantes, e concluíssem que o gráfico de uma função quadrática qualquer é um deslocamento horizontal ou vertical do gráfico de uma função do tipo $f(x)=a x^{2}$, em que $a$ é uma constante não nula. 


\section{Descrição da Atividade}

A atividade foi realizada na escola privada durante as aulas de matemática. Os alunos foram orientados a baixar o aplicativo GeoGebra no celular (figura 1), seguir um roteiro de construçōes, investigar a partir de perguntas realizadas no decorrer da atividade, acompanhar a demonstração da fórmula de Bhaskara no quadro e responder a um questionário que procurava saber suas impressôes acerca do uso do GeoGebra no estudo de funçôes.

Figura 1 - Alunos utilizando o aplicativo na escola privada.

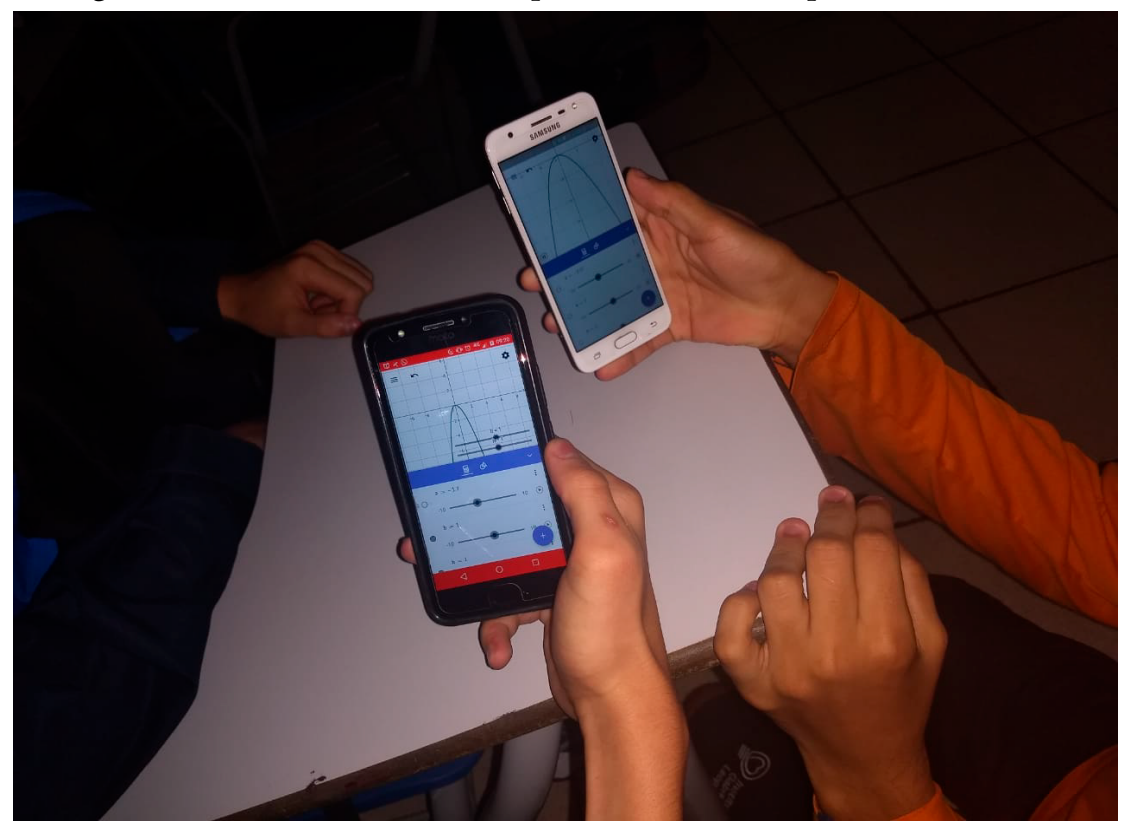

Fonte: autores

Nossos objetivos com a atividade foram levar os alunos a:

verificar que o gráfico de qualquer função quadrática da forma $j(x)=$ $a x^{2}+b x+c$ pode ser obtido através de deslocamentos horizontais e verticais do gráfico de $f(x)=a x^{2}$;

1. utilizar o fato anterior para demonstrar a fórmula de Bhaskara. 


\section{Roteiro da atividade no GeoGebra}

1. Criar controles deslizantes para os parâmetros $a, k$ e $h$, com intervalo entre -10 e 10 e incremento 0,01 ;

2. Na caixa de entrada, criar a função $f(x)=a x^{2}$;

3. Variar o parâmetro $a$ e observar o que acontece com a concavidade da parábola;

4. Na caixa de entrada, criar a função $g(x)=a(x-k)^{2}$;

5. Variar o parâmetro $\mathrm{k}$ e observar o deslocamento horizontal da parábola (fig. 3);

6. Na caixa de entrada, criar a função $l(x)=a(x-k)^{2}+b$;

7. Variar o parâmetro $h$ e observar o deslocamento vertical da parábola (fig. 4);

8. Criar os controles deslizantes $b$ e $c$, o gráfico de $j(x)=a x^{2}+b x+c$ e ajustar $h$ e $k$, através dos deslocamentos, no sentido de coincidir os gráficos de $j(x)$ e $l(x)$;

Figura 2 - Gráfico das funçóes $f(x)$ e $g(x)$

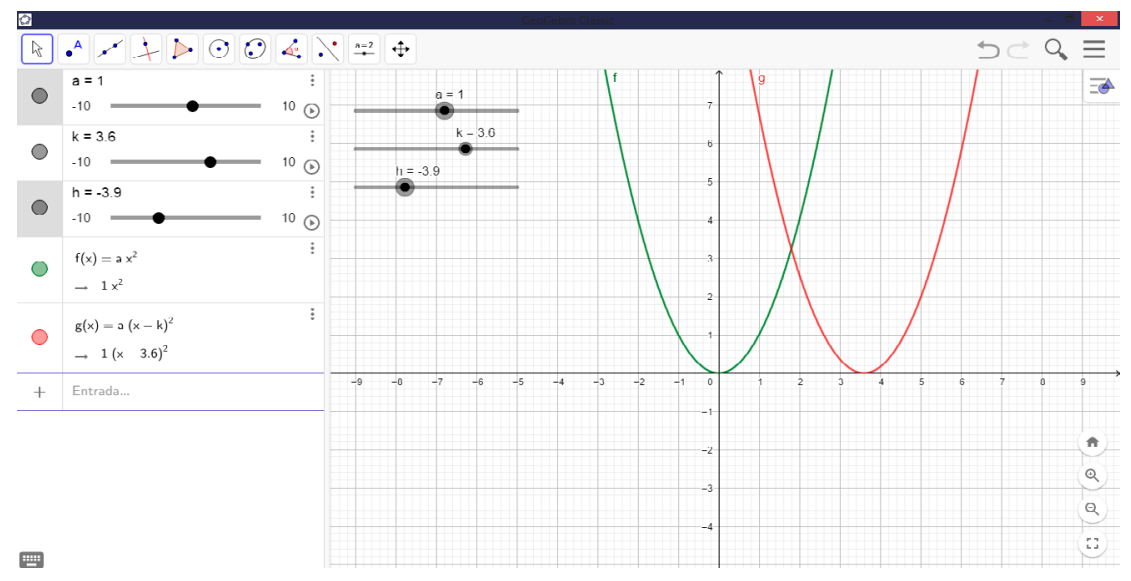

Fonte: autores 
Figura 3 - Gráfico das funçôes $f(x)$ e $l(x)$

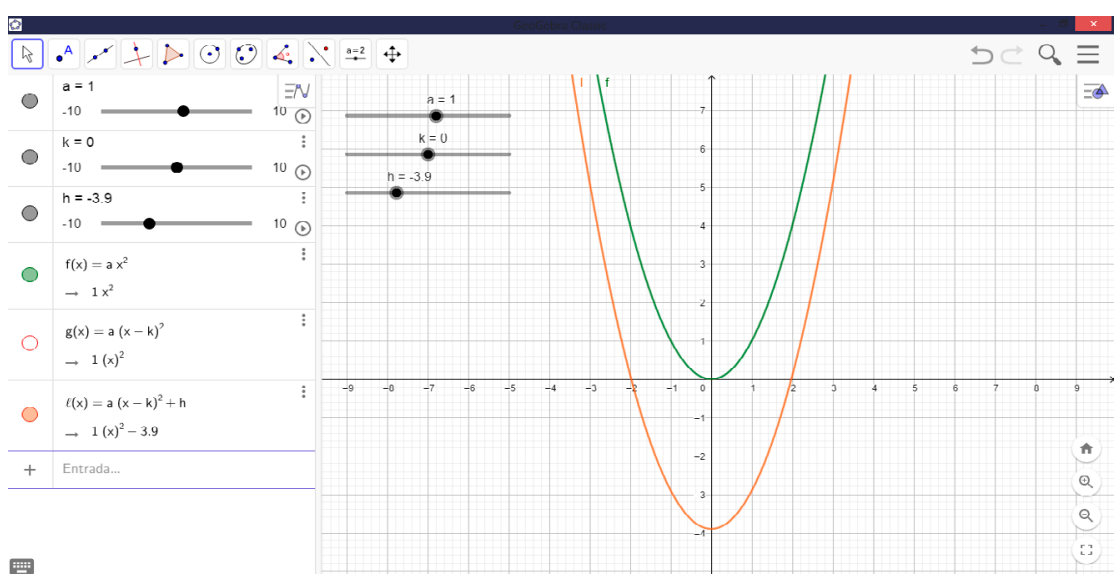

Fonte: autores estudantes.

Durante a realização da atividade, fizemos algumas perguntas aos

1. Que relação existe entre os gráficos das funções $f(x)$ e $g(x)$ ?

2. Que relação existe entre os gráficos das funçóes $f(x)$ e $l(x)$ ?

3. Como podemos determinar as raízes da função $l(x)$ ?

4. Como podemos escrever $a(x-k)^{2}+h=0$ na forma $a x^{2}+b x+$ $c=0$ ?

5. Qual é o valor do coeficiente $b$ e do coeficiente $c$ no item anterior?

6. Como podemos determinar os valores dos parâmetros $k \mathrm{e} h$ ?

7. Os valores de $k$ e $h$ se assemelham com algo que vocês já estudaram?

8. Como podemos obter a fórmula de Bhaskara a partir de $l(x)=$ $a(x-k)^{2}+h=0$ ?

9. Como podemos obter a fórmula de Bhaskara completando quadrados?

\section{Demonstração da fórmula de Bhaskara}

$\mathrm{Na}$ condução da atividade, os estudantes puderam concluir que, dado o gráfico da função $j(x)$, era possível ajustar os parâmetros $k$ e $h$ de modo que o gráfico da função $l(x)$ coincidisse com o de $j(x)$, isto é, o gráfico da função 
$j(x)$ é obtido através de deslocamentos horizontais e verticais do gráfico da função . Isso nos conduz a concluir que existe uma relação entre $k$ e $h$ e os parâmetros $a, b$ e $c$. Esse foi o norte para realizar a demonstração seguindo o roteiro de perguntas apresentado acima.

Iniciamos a demonstraçáo com a seguinte pergunta: como podemos determinar as raízes da função $l(x)=a(x-k)^{2}+h$ ? Para determinar as raízes da função, devemos tomar $l(x)=0$. Realizando-se as manipulaçóes algébricas necessárias, encontra-se

$$
x=k \pm \sqrt{-\frac{h}{a}}
$$

Perguntamos, em seguida, como podemos escrever $a(x-k)^{2}+h=0$ na forma $a x^{2}+b x+c=0$. Para isso, basta desenvolver a expressão $a(x-k)^{2}$ $+h=O$ e obter

$$
\mathrm{ax}^{2}-2 \mathrm{akx}+\mathrm{ak}^{2}+h=0
$$

Na sequência, perguntamos: Qual é o valor dos coeficientes $b$ e $c$ em (2)? Comparando a equação (2) com a equação $a x^{2}+b x+c=0$, podemos concluir que:

$$
b=-2 \mathrm{ake} c=\mathrm{ak}^{2}+h
$$

Seguimos a demonstração questionando como podemos determinar os valores dos parâmetros $k$ e $h$ em termos de $a, b$ e $c$. Por (3), percebe-se que

$$
k=-\frac{b}{2 a}
$$

$$
h=c-\mathrm{ak}^{2}=c-a\left(\frac{b^{2}}{4 a^{2}}\right), \text { o que implica em } h=\frac{4 \mathrm{ac}-b^{2}}{4 a}, \text { ou seja }
$$

$$
h=-\frac{\Delta}{4 a}, \text { em que } \Delta=b^{2}-4 \text { ac. }
$$

Com isso, os estudantes puderam perceber que $k$ e $h$ são, na verdade, as coordenadas de $x$ vértice e $y$ vértice, respectivamente. Alguns alunos não reconheceram o valor de $h$ como a coordenada $y$ vértice. Ao estudarem o 
conteúdo, os alunos aprenderam a substituir a coordenada $x$ vértice na função para encontrar a coordenada $y$.

Para finalizar a demonstração, perguntamos como podemos obter a fórmula de Bhaskara a partir dos resultados anteriores. A fórmula de Bhaskara é obtida substituindo as expressóes (4) e (5) em (1). Realizando as manipulações algébricas necessárias, obtém-se

$$
x=\frac{-b \pm \sqrt{\Delta}}{2 a} .
$$

\section{Análise}

Dos 28 estudantes sujeitos deste estudo, nenhum havia aprendido o conteúdo de funçóes quadráticas, total ou parcialmente, por meio de tecnologia digital. ${ }^{5}$ A demonstração da fórmula de Bhaskara, quando ocorreu, utilizou o método tradicional de completar quadrados.

O uso de smartphone para aprender esse conteúdo era uma novidade para todos os estudantes. Ele funcionou como instrumento mediador da aprendizagem, pois se interpôs entre o estudante e o objeto matemático, alterando a maneira como isso se daria, comparado a uma aula convencional - ou seja, o smartphone foi dirigido para o controle externo. Por outro lado, ele teve papel de signo. Os estudantes, ao se apropriarem desse novo uso de seus aparelhos, não os viam mais somente como objetos para fazer ligaçóes e acessar redes sociais. Ao olharem para seus smartphones e pelo vivido no social (experiência na atividade), os aparelhos teriam, para eles, uma nova representação mental e subjetiva: o estudo.

O GeoGebra, por sua vez, representado pelos seus comandos, funcionou como signo, dirigido à atividade interna do sujeito. Após a execução do comando do roteiro, na caixa de entrada criar a função $f(x)=a x^{2}$, por exemplo, $f(x)=a x^{2}$ tornou-se, no plano interno, uma parábola com vértice na origem. Da mesma forma, o parâmetro $\mathrm{k}$, no plano interno, passou a se relacionar com o deslocamento horizontal da parábola.

Todas essas novas aquisiçóes foram possíveis pelo uso das tecnologias digitais. Seria bastante difícil ao professor, utilizando somente o quadro,

5 Dado obtido das respostas à pergunta: quando você estudou funções quadráticas, seu professor(a) utilizou algum recurso computacional? 
dar à aula esse dinamismo. A forma interativa proporcionada pelo uso do GeoGebra é dinâmica, plástica, atrativa e permite autonomia (os estudantes, embora com o roteiro, tinham liberdade para manipular parâmetros, por exemplo). Isso está em consonância com as ideias de Levy (1993) quando ele considera que

(u)m modelo digital não é lido ou interpretado como um texto clássico, ele geralmente é explorado de forma interativa. Contrariamente à maioria das descriçóes funcionais sobre o papel ou aos modelos reduzidos analógicos, o modelo informático é essencialmente plástico, dinâmico, dotado de uma certa autonomia de ação e reação. (LEVY, 1993, p. 121).

A busca pelo conhecimento se deu de forma diferente para os sujeitos do estudo. $\mathrm{O}$ acesso aos gráficos durante a manipulação do GeoGebra, a liberdade para experimentar, a facilidade de visualizaçáo e a maneira como se deu a relação entre professores e estudantes, mostram uma forma diferente de organizar/reorganizar o pensamento. Este foi produzido como resultado de um processo interativo entre professor, estudantes, smartphone e GeoGebra, em um coletivo pensante seres-humanos-com-GeoGebra-e-smartphone. Todos os atores envolvidos, humanos e náo humanos, tiveram papel crucial ao longo da atividade.

Embora possa parecer que o papel do professor, ao demonstrar a fórmula de Bhaskara, não contribuiu qualitativamente para reorganizar o pensamento dos estudantes, vale ressaltar que ele o fez de modo muito diferente do que é o habitual, um caminho que levou o estudante a mudar a forma de compreender o conteúdo. A fórmula de Bhaskara não foi somente apresentada aos estudantes. Eles trilharam um caminho por meio do qual puderam compreender, de forma coerente e acessível, a demonstração feita pelo professor. Nota-se, portanto, que nesse coletivo, todos assumiram papel importante. Em vários momentos, o professor assumiu o papel de mediador da aprendizagem, interpondo-se entre os estudantes e o objeto matemático. Este papel está em sintonia com as palavras de Carneiro e Passos (2014, p. 102), quando as autoras afirmam que "o professor precisa participar de forma ativa do processo de construçáo do conhecimento do aluno, sendo um mediador, motivador e orientador da aprendizagem”. Uma resposta de um dos estudantes traduz bem a importância do GeoGebra e do professor como mediadores: 
$\mathbf{A}_{6}$ : [...] a explicação do professor, conciliada com o uso do GeoGebra, será ideal para o entendimento mais completo possível do aluno.

As ideias contidas nos últimos parágrafos estáo relacionadas à concepção de reorganização de pensamento de Tikhomirov (1981), que a associa à geraçáo de um estágio de pensamento qualitativamente diferente, que reorganiza os processos de criação, busca e armazenamento de informação, e as relaçóes humanas.

Algumas respostas dos estudantes à pergunta "o que você achou da atividade desenvolvida?" fornecem pistas da reorganizaçăo do pensamento, na perspectiva de Thikomirov (1981). Em relação aos processos de criaçáo, busca e armazenamento de informaçóes, por exemplo, foram citados avanços qualitativos relacionados à interatividade, dinamismo, facilidade para aprender. Relacionadas ao dinamismo e à interatividade, apresentamos duas respostas.

$\mathbf{A}_{1}$ : A atividade foi bastante interativa ${ }^{6}$

$\mathbf{A}_{2}$ : Produtiva e mais dinâmica

O dinamismo e a interatividade do GeoGebra permitiram aos estudantes percorrerem um caminho não linear na construção do conhecimento. Eles podiam, por exemplo, gerar um gráfico com rapidez, apagá-lo, alterá-lo, voltar a um gráfico anteriormente apagado, considerar uma sugestáo do professor e executar um novo comando, experimentar novos comandos, etc. Essas possibilidades oferecidas pelo GeoGebra desafiam a linearidade do pensamento ao aliar oralidade, visualizaçáo, experimentaçáo. Os estudantes se apropriaram dos conhecimentos, utilizando elementos mediadores diferentes dos comumente utilizados. Além do professor, do lápis e do papel, outros mediadores compuseram o coletivo pensante, possibilitando uma mudança qualitativa no tipo de apropriação do conhecimento.

Relacionadas à atividade como facilitadora da aprendizagem, apresentamos três respostas: conteúdo.

$\mathbf{A}_{3}$ : A atividade foi excelente e auxilia na melhor compreensão do

$\mathbf{A}_{4}$ : Eu acho interessante o uso do GeoGebra, pois consegui perceber melhor o que ocorre no valor das incógnitas demonstradas.

$\mathbf{A}_{5}$ : A atividade e o uso do GeoGebra ajudaram a entender mais.

$\mathbf{A}_{27}$ : Entendi muito mais rápido do que no quadro negro.

6 As respostas foram transcritas para mantê-las exatamente como foram dadas. 
Acreditamos que a compreensão mais fácil é resultado da criação de um coletivo que ofereceu aos estudantes novas possibilidades de organizar o pensamento. Vários estudantes disseram se sentir mais motivados para aprender, após a atividade.

$\mathbf{A}_{3}$ : me deixou mais motivado.

$\mathbf{A}_{21:}$ nos deixa mais motivados e com vontade de participar, pois foge daquela realidade de quadro.

$\mathbf{A}_{22}$ : o uso do GeoGebra no estudo de conteúdos matemáticos me deixa mais motivado, pois é uma nova forma de estudo.

A partir dessas respostas, acreditamos que o uso do smartphone e do GeoGebra constitui-se como aquilo que a literatura relativa à motivação denomina de embelezamento motivacional (BZUNECK, 2010), em estratégias de ensino que contribuem para se conseguir melhor envolvimento dos alunos nas atividades de aprendizagem. De acordo com Bergin (1999), apud Bzuneck (2010), um dos tipos de embelezamento motivacional é a introdução de novidades, o que parece se encaixar no caso da atividade desse estudo. Chamar a atenção para o aspecto motivacional nas relaçóes estabelecidas dentro do coletivo é importante, já que a aprendizagem não depende apenas de capacidade cognitiva, mas, sim, de uma "interação entre cognição, motivação e afeto" (SOUZA, 2006, p. 111).

\section{Consideraçóes finais}

Neste estudo, nosso objetivo foi desvelar apropriaçóes de novos conhecimentos relacionados à demonstração da fórmula de Bhaskara num coletivo seres-humanos-com-GeoGebra-e-smartphone e apresentar indícios de pensamento reorganizado.

Os resultados mostraram que o uso de novos mediadores, como GeoGebra e smartphone, além de o professor exercendo papel de auxiliar no processo de aprendizagem, dando liberdade e autonomia aos estudantes, permitiu apropriaçáo de novos conhecimentos em relação à demonstraçáo da fórmula de Bhaskara. $\mathrm{O}$ uso de instrumentos de signos permitiu a apropriação de novos caminhos para a aprendizagem, por meio da experimentação, visualização e, sobretudo, de uma forma de aprendizagem não linear, diferente daquela que comumente ocorre em sala de aula. Acreditamos que isso pode ter, em alguma medida, contribuído para a reorganização do pensamento dos estudantes. 
Além disso, o estudo mostra a importância de compreendermos a aprendizagem como resultado de açóes dentro de um coletivo, dentro do qual todos "aprendem", atores humanos e não humanos. Isso corrobora as ideias de Levy (1993), para quem as tecnologias não são neutras ou transparentes, e tampouco devem ser apenas adereço ou maquiagem. Elas são, na verdade, parte constituinte de um coletivo pensante.

\section{Referências}

BORBA, M. C.; SCUCUGLIA, R. R. S.; GADANIDIS, G. Fases das tecnologias digitais em Educação Matemática: sala de aula e internet em movimento. Belo Horizonte: Autêntica, 2014.

BORBA, M. C.; VILLARREAL, M. Humans-with-media and the reorganization of mathematical thinking: information and communication technologies, modeling, visualization and experimentation. USA: Springer, 2005.

BORBA, M.C. Coletivos seres-humanos-com-mídias e a produção matemática. In: I Simpósio Brasileiro de Psicologia da Educação Matemática. Anais I Simpósio, Curitiba, 2001.

BZUNECK, J. A. Como motivar os alunos: sugestóes práticas. In: BORUCHOVITCH, E; BZUNECK, J. A.; GUIMARÁES, S. E. R. (org.). Motivação para aprender: aplicaçôes no contexto educativo. Petrópolis, RJ: Editora Vozes, 2010. p. 13-42.

CARNEIRO, R. F.; PASSOS, C. L. B. A utilização das Tecnologias da Informação e Comunicação nas aulas de Matemática: Limites e possibilidades. Revista Eletrônica de educação, São Carlos, v. 8, n. 2, p. 101$119,2014$.

LEVY, P. As tecnologias da inteligência: o futuro do pensamento na era da informática. Rio de Janeiro: Editora 34, 1993.

PINO, A. A mediação semiótica em Vygotsky e seu papel na explicação do psiquismo humano. Caderno Cedes. Pensamento e linguagem: estudos na perspectiva da psicologia soviética, Campinas, n. 24, p. 32-43, 1991. 
RIPPER, A. V. Significação e mediação por signo e instrumento. Temas psicol. Ribeirão Preto, v. 1, n. 1, p. 25-30, abr. 1993. Disponível em http:// pepsic.bvsalud.org/scielo.php?script=sci_arttext\&pid=S1413-389X199300 0100005\&lng=pt\&nrm=iso. Acesso em: 05 maio 2020.

SMOLKA, A. L. B. O (im)próprio e o (im)pertinente na apropriação das práticas sociais. Caderno Cedes, Campinas, v. 20, n. 50, p. 26-40. 2000.

SOUZA, L. N. I. Crenças de autoeficácia matemática. In: AZZI, R. G.; POLYDORO, S. A. J. (org.). Autoeficácia em diferentes contextos. Campinas: Alínea, 2006. p. 111-126.

TIKHOMIROV, O. K. The psychological consequences of the computerization. In: WERSTCH, J. (ed.), The concept of activity in soviet psychology. New York: Sharp, 1981. p. 256-278.

TORISU, E. M. (2018). Sentidos e significados atribuídos por um grupo de estudantes às tarefas matemáticas. Zetetike, Campinas, v. 15, n. 2, p. 390-403, 2018.

VERASZTO, E. V. et al. Tecnologia: buscando uma definição para o conceito. Prisma.com, Porto, n. 7, p. 60-84, 2008.

VIGOSTSKY, L. S. Concret human Psychology. Soviet Psychology, v. 27, n. 2, p. 53-77, 1989.

Recebido em: 24 ago. 2019.

Aceito em: 23 mar. 2020. 